\title{
Biochemistry and Pharmacology Cancer Activity
}

National Cancer Institute

\section{Source}

National Cancer Institute. Biochemistry and Pharmacology Cancer Activity. NCI

Thesaurus. Code C19267.

Preclinical studies designed to improve cancer treatment, with an emphasis on the discovery of new drugs and treatment strategies, selective targeting, development of new preclinical models, and understanding, preventing, and overcoming drug resistance. 